\title{
Rechtliche und technische Nutzerunterstützung bei der Button-Lösung
}

\author{
Carina Boos, LL.M., Kassel / Dr. Steffen Bartsch, Darmstadt / Prof. Dr. Melanie Volkamer, \\ Darmstadt
}

Durch die im August 2012 eingeführte sogenannte Button-Lösung soll der Verbraucher die rechtliche Situation bei Kostenfallen - also das Nichtzustandekommen des Vertrags - einfacher erkennen und seine Position deshalb auch selbstbewusst gegenüber dem Unternehmer vertreten können.

In diesem Beitrag wird zunächst die Idee der Nutzerunterstützung gegen Kostenfallen erläutert (I.), bevor die Button-Lösung detailliert aus rechtlicher Sicht dargestellt wird und Unterschiede zur früheren Rechtslage aus Verbrauchersicht aufgezeigt werden (II.). Daraus lassen sich weiterhin bestehende Probleme für den Verbraucher ableiten (III.). Im Bewusstsein dieser Probleme wird untersucht, wie der Verbraucher zusätzlich zu den verbraucherrechtlichen Schutzbestimmungen durch technische Maßnahmen unterstützt werden kann. Dazu wird erörtert, in welchem Umfang es möglich ist, den Nutzer mit entsprechender Software auf dessen Gerät zu unterstützen. Im Fokus steht dabei das automatisierte Erkennen von Webseiten, durch die der Verbraucher einen ihn zur Zahlung verpflichtenden Vertrag abschließen kann. Zur Unterstützung sollen etwa gezielte Hinweise genutzt werden (IV.). Die Ergebnisse dieser Überlegungen werden schließlich in einem Fazit zusammengefasst (V.).

\section{Nutzerunterstützung gegen Kostenfallen}

Im ständig weiter wachsenden Online-Handel sind neben vielen seriösen Anbietern auch solche vertreten, die die Besucher ihrer Webseite „abzocken“. Bei sogenannten Kostenfallen merkt der Verbraucher häufig nicht, dass er mit dem Anbieter vermeintlich einen Vertrag abschließt, der eine Zahlungspflicht begründen soll. Er gibt seine persönlichen Daten an, um einen im Internet regelmäßig kostenlos angebotenen Dienst zu nutzen, ohne dass er auf die anfallenden Kosten für die Nutzung aufmerksam gemacht wird. Erst im Nachhinein erhält er eine Rechnung, die ihn die Kosten bemerken lässt. ${ }^{1}$ Viele Verbraucher begleichen solche Rechnungen sofort oder zahlen, nachdem sie durch wiederholte (auch anwaltliche) Zahlungsaufforderungen eingeschüchtert wurden. Diese Verbraucher gehen davon aus, dass sie etwas übersehen oder versehentlich angeklickt und damit zugestimmt haben. Sie zahlen, weil sie sich über die Rechtslage im Unklaren sind oder wegen der meist geringen Summen den (auch emotionalen) Aufwand einer Auseinandersetzung und mögliche Gerichtsverhandlungen scheuen. $^{2}$

Um den Verbraucher vor unseriösen Anbietern zu schützen und dabei zu unterstützen, kostenpflichtige Dienste zu erkennen, wurde zum 1. August 2012 die sogenannte „ButtonLösung“ in $\S 312 \mathrm{~g}$ BGB eingeführt. Dabei handelt es sich um eine Vorabumsetzung des Art. 8 Abs. 2 Verbraucherrechterichtlinie ${ }^{3}$. Danach kommt ein Vertrag, der eine Zahlungspflicht begründet, nur zustande, wenn der Unternehmer bestimmte Informations- und Gestaltungspflichten eingehalten hat. Dem Verbraucher wird es ohne nähere Kenntnisse der Rechtslage - gerade bei gewollt unklarer Gestaltung, beispielsweise der Verwendung einer nicht vom Gesetzgeber genannten Beschriftung - dennoch immer noch nicht möglich sein, die

Vgl. etwa Meyer-van Raay/Deitermann, VuR 2009, 335, 335 f.

BT-Drs. 17/7745, S. 6.

Richtlinie 2011/83/EU des Europäischen Parlaments und des Rates vom 25. Oktober 2011 über die Rechte der Verbraucher, zur Abänderung der Richtlinie 93/13/EWG des Rates und der Richtlinie 1999/44/EG des Europäischen Parlaments und des Rates sowie zur Aufhebung der Richtlinie 85/577/EWG des Rates und der Richtlinie 97/7/EG des Europäischen Parlaments und des Rates vom 25.10.2011, ABl. EU vom 22.11.2011, Nr. L 304, S. 64. Zum 13. Juni 2014 wird die übrige Umsetzung der Verbraucherrechterichtlinie im BGB und EGBGB in Kraft treten. 
Rechtskonformität mit Sicherheit zu bestimmen. ${ }^{4}$ Deshalb bietet sich eine technische Unterstützung des Verbrauchers an, die dort anknüpft, wo das Recht seine Grenzen findet - bei der individuellen Unterstützung des Verbrauchers in einer konkreten Situation.

Dazu könnte eine Software genutzt werden, die der Verbraucher auf seinem Gerät installiert und die ihn darauf aufmerksam macht, sobald eine Webseite eine - zumindest vermeintlich den gesetzlichen Vorgaben entsprechende - Schaltfläche (häufig als „Button“ bezeichnet) enthält. Damit könnte der Verbraucher zum einen erkennen, ob er im Begriff ist, einen Vertrag abzuschließen, der eine Zahlungspflicht seinerseits begründet, und zum anderen, ob er dabei ist, einer Kostenfalle zu erliegen. Beides könnte den Verbraucher davor bewahren, einen ungewünschten, ressourcenverbrauchenden Prozess $\mathrm{zu}$ führen oder bereits im Vorfeld unberechtigte Zahlungsansprüche zu erfüllen.

\section{Gesetzlicher Rahmen}

Will ein Unternehmer einen Vertrag über die Lieferung von Waren oder über die Erbringung von Dienstleistungen mit einem Verbraucher über seine Webseite abschließen, muss er seit dem letzten Jahr zusätzliche rechtliche Bestimmungen einhalten, wenn mit dem Vertragsabschluss eine Zahlungspflicht des Verbrauchers verbunden ist. Die kürzlich beschlossene und zum Juni 2014 in Kraft tretende Umsetzung der ,übrigen“ Verbraucherrechterichtlinie wird diese Rechtslage nicht erneut verändern. ${ }^{5}$

Neben der Frage, welche konkreten Anforderungen sich aus der Gesetzesänderung ergeben, ist auch zu klären, ob sich durch die Gesetzesänderung die Lage für den Verbraucher verbessert.

\section{Anforderungen an Webseiten nach $§ 312 \mathrm{~g}$ Abs. 3 BGB}

Um zu untersuchen, inwieweit zusätzlich zu der gesetzlich bestimmten Button-Lösung eine Unterstützung des Verbrauchers nötig und möglich ist, müssen zunächst die Anforderungen zusammengetragen werden, die sich aus $\S 312 \mathrm{~g}$ Abs. 3 BGB ergeben. ${ }^{6}$ Auch die wenige bisher zu dieser Norm ergangene Rechtsprechung wird dabei herangezogen. Vom Anbieter wird eine bestimmte Gestaltung der Webseite gefordert. Verstößt der Unternehmer gegen diese, ist die Konsequenz, dass der die Zahlungspflicht begründende Vertrag nach Abs. 4 nicht zustande kommt. ${ }^{7}$

$\S 312 \mathrm{~g}$ Abs. 3 Satz 1 BGB bestimmt zunächst technikneutral ${ }^{8}$ und abstrakt für alle Vertragsabschlüsse zwischen Unternehmern und Verbrauchern im elektronischen Geschäftsverkehr, dass der Bestellvorgang vom Unternehmer so gestaltet werden muss, „,dass der Verbraucher mit seiner Bestellung ausdrücklich bestätigt, dass er sich zu einer Zahlung verpflichtet.“ Dies

4 Auch großen Online-Unternehmen wie Amazon war es nicht ohne weiteres möglich, die gesetzlichen Pflichten, die sich aus der Button-Lösung ergeben, umzusetzen. Vgl. Höwelkröger, Verstoß gegen „ButtonLösung“: Einstweilige Verfügung gegen Amazon, heise resale vom 26.7.2013, http://www.heise.de/1924735.html, letzter Abruf am 9.8.2013.

$5 \S 312 \mathrm{~g}$ Abs. 3 und 4 BGB werden ab dem 13. Juni 2014 wortgleich in $\S 312 \mathrm{j}$ Abs. 3 und 4 BGB n. F. übernommen. (Die einzigen Veränderungen ergeben sich aufgrund geänderter Verweisungen.)

$6 \quad$ Vgl. auch Bierekoven, ITRB 2012, 186, 187; Ernst, VuR 2012, 205, 205. Zum Teil wird auch Abs. 2 unter den Begriff der Button-Lösung gezählt; vgl. etwa Leier, CR 2012, 378, 381 f.; Wilschke, VuR 2012, 171, 172. Da dieser lediglich die Wiederholung bestimmter Informationspflichten vorsieht, sich nicht direkt auf die Schaltfläche bezieht und ein Verstoß andere Rechtsfolgen nach sich zieht, soll er im Rahmen dieses Beitrags nicht detailliert betrachtet werden.

7 Die rechtliche Einordnung dieser Norm ist umstritten. Für eine Formvorschrift und damit rechtshindernde Einrede etwa Raue, MMR 2012, 438; für eine Vermutungsregelung durch teleologische Reduktion etwa Kirschbaum, MMR 2012, 8; für eine teleologische Reduktion, wenn sich der Unternehmer auf Abs. 4 beruft, und vertraglichen Schadenersatz Weiss, JuS 2013, 590, 593 f.

$8 \quad$ Vgl. Leier, CR 2012, 378, 383; Grüneberg, in: Palandt, BGB, 72. Aufl. 2013, § 312g BGB, Rn. 14. 
gilt etwa beim massenhaften Versand von Werbe-E-Mails mit der Möglichkeit der Bestellung durch Beantworten der Mail.

Für den im Folgenden betrachteten, faktisch besonders relevanten Fall, dass die Bestellung über eine Schaltfläche erfolgt, mit der etwa ein Online-Bestellformular abgesendet wird, nennt Satz 2 konkretere Anforderungen. Dabei ist der Begriff Schaltfläche nicht ausschließlich im Sinne eines Buttons zu verstehen, sondern erfasst nach der Gesetzesbegründung ,jedes grafische Bedienelement, das es dem Anwender erlaubt, eine Aktion in Gang zu setzen oder dem System eine Rückmeldung zu geben.“ Dazu zählen auch Bedienelemente mit einer ,ähnliche(n) Funktion wie ein Bedienknopf", wie ein Hyperlink oder eine Checkbox. ${ }^{9}$ Eine Pflicht, eine solche Schaltfläche für den Vertragsabschluss vorzusehen, wird nicht festgeschrieben. Dies ist aber auch nicht nötig, da sämtliche Optionen erfasst werden, durch die ein Vertrag innerhalb eines Online-Shops geschlossen werden kann. ${ }^{10}$

\section{a) Beschriftung der Schaltfläche}

Um den Anforderungen des $\S 312 \mathrm{~g}$ Abs. 3 Satz 2 BGB zu genügen, muss die Schaltfläche zur Erklärung der rechtsverbindlichen Willenserklärung des Verbrauchers mit „den Wörtern ,zahlungspflichtig bestellen " oder mit einer entsprechend eindeutigen Formulierung beschriftet" sein. Entscheidend ist, dass für den Verbraucher allein durch die Betrachtung der Schaltfläche erkennbar wird, dass er sich mit dem Anklicken derselben zu einer Zahlung verpflichtet. ${ }^{11}$

\section{aa) Rechtskonforme Beschriftungen}

Neben der Beschriftung ,zahlungspflichtig bestellen“ sind weitere rechtskonforme Möglichkeiten zur Beschriftung der Schaltfläche nach der Gesetzesbegründung ,kostenpflichtig bestellen“ und „zahlungspflichtigen Vertrag schließen“. Abhängig davon, wie und welcher Vertragstyp abgeschlossen wird, sind auch die Beschriftungen „kaufen“ und bei OnlineAuktionen „Gebot abgeben“ oder „Gebot bestätigen“" zulässig. ${ }^{12}$ Die Art der Webseite und des Vertrags kann damit spezielle Beschriftungen rechtfertigen. In der Literatur wird außerdem die Beschriftung „Vertrag schließen (kostenpflichtig)“ und ,jetzt kaufen“ als zulässig eingeordnet. ${ }^{13}$ Nicht ausreichend eindeutig sind nach der Gesetzesbegründung die Beschriftungen „Anmeldung“, „weiter“, „,bestellen“, „Bestellung abgeben“ oder „Bestellung abschließen“. ${ }^{4}$

Aufgrund des gesetzgeberischen Ziels - dem Schutz der Verbraucher durch klare, leicht verständliche Bestimmungen - müssen sonstige Beschriftungen kritisch geprüft werden, um sicherzustellen, dass der Verbraucher eindeutig auf die möglicherweise entstehende Zahlungspflicht hingewiesen wird. Auch das LG Berlin legte einen scharfen Maßstab an, als es die Beschriftung ,Jetzt verbindlich anmelden! (zahlungspflichtiger Reisevertrag)“ als unzulässig bewertete. Die Bezeichnung ,anmelden“ deute darauf hin, dass das Anklicken der Schaltfläche lediglich eine „Vorbereitungshandlung“ darstelle. Daran konnte auch die Ergänzung ,zahlungspflichtiger Reisevertrag“ und ,verbindlich“ nichts ändern. ${ }^{15}$

9 BT-Drs. 17/7745, S. 12

10 So auch Leier, CR 2012, 378, 383; wohl auch Raue, MMR 2012, 438, 438; unsicher Roth, VuR 2012, 477 , 481.

11 Zwar wurde etwa von Schwartmann, Stellungnahme vor dem Rechtsausschuss des Deutschen Bundestages, 6.2.2012, http://www.bundestag.de/bundestag/ausschuesse17/a06/anhoerungen/archiv/17_Kostenfallen/ 04_Stellungnahmen/Stellungnahme_Schwartmann.pdf, S. 6, letzter Abruf am 9.8.2013, kritisiert, dass die Zahlungspflicht erst nach Vertragsschluss besteht, also erst nach Annahme des Vertrags, die regelmäßig im Nachhinein durch den Unternehmer erfolgt. Es ist jedoch durchaus zweckmäßig, den Verbraucher dann auf die (vermeintliche) Zahlungspflicht hinzuweisen, bevor er seine rechtsverbindliche Willenserklärung abgibt, da er danach keinen Einfluss mehr darauf nehmen kann, ob es zum Vertragsschluss kommt.

12 BT-Drs. 17/7745, S. 12.

13 Raue, MMR 2012, 438, 438.

14 BT-Drs. 17/7745, S. 12.

15 LG Berlin, Urteil vom 17.7.2013, AZ: 97 O 5/13. 
Es kann jedoch aus Platzgründen beispielsweise bei mobilen Webseiten nötig sein, kurze Beschriftungen, wie ein einzelnes „€“-Symbol zu verwenden. ${ }^{16}$ Bei entsprechender formaler Gestaltung der Schaltfläche kann auch dies ausreichen, um auf die mögliche Zahlungspflicht aufmerksam zu machen. ${ }^{17}$

\section{bb) Beschriftung ,mieten“"}

Eine Beschriftung mit „mieten“ oder ,,jetzt mieten“ kann hingegen nicht ausreichen, um den durchschnittlichen Verbraucher auf die Kostenpflichtigkeit hinzuweisen. Eine solche Formulierung würde sich jedoch gerade im Hinblick auf das Cloud Computing anbieten, da Verträge insbesondere über die Nutzung klassischer Cloud-Dienste als Mietverträge eingeordnet werden können. ${ }^{18}$ Betreiber von Kostenfallen könnten etwa die sonst häufig kostenlose Nutzung von Cloud-Speicherdiensten zahlungspflichtig anbieten und die Schaltfläche - analog zu „kaufen“ bei Online-Shops - mit „mieten“ beschriften.

Mieten i.S.d. § 535 Abs. 2 BGB impliziert, dass der Mieter Miete zahlen muss. Werden Sachen hingegen unentgeltlich zum Gebrauch überlassen, handelt es sich um Leihe i.S.d. § 598 BGB. Diese juristisch präzise Unterscheidung spiegelt sich jedoch nicht im allgemeinen Sprachgebrauch wider. Dort wird häufig nicht klar zwischen den Begriffen der Leihe und Miete unterschieden. Zwar wird vor allem der Begriff der Leihe anstelle der Miete verwendet. ${ }^{19}$ Aber auch die Kombination „kostenlos mieten“ wird im alltäglichen Sprachgebrauch verwendet und würde einen juristisch nicht vorgebildeten Laien, wie es Verbraucher i.d.R. sind, nicht verwundern. Dem Verbraucher ist die juristische Einordnung eines Vertrags in die Vertragstypologien des BGB regelmäßig unbekannt.

Anders als beim Begriff „kaufen“ ist dem durchschnittlichen Verbraucher bei Verwendung des Begriffs „mieten“ deshalb nicht intuitiv klar, dass es sich um einen Vertrag handelt, der eine Zahlungspflicht begründet. In Anbetracht des allgemein undifferenzierten Sprachgebrauchs ist allein der Begriff „mieten“ nicht ausreichend, um auf die Zahlungspflicht hinzuweisen. Potential für eine Formulierung, die der Verbraucher nicht als eindeutig einordnet, besteht demnach auch nach Einführung der Button-Lösung.

\section{cc) Schaltflächen mit Beschriftung}

Im Übrigen muss im Umkehrschluss aus $\S 312 \mathrm{~g}$ Abs. 3 BGB gefolgert werden, dass lediglich die Schaltfläche, die final die rechtsverbindliche Willenserklärung des Verbrauchers übermittelt, mit den genannten Begriffen beschriftet werden darf. ${ }^{20}$ Würden andere Schaltflächen entsprechend beschriftet, müsste der Verbraucher davon ausgehen, dass er bereits ab diesem Zeitpunkt an seine Erklärung gebunden ist.

\section{dd) Beschriftungszusätze}

Neben der unmissverständlichen Formulierung darf die Schaltfläche mit keinen ablenkenden oder irreführenden Zusätzen versehen werden. ${ }^{21}$ Dies umfasst zunächst die Beschriftung des Buttons, die sich auf die zuvor genannten oder entsprechend eindeutigen Bezeichnungen beschränken muss. So wurde etwa „Jetzt verbindlich anmelden! (zahlungspflichtiger Reisevertrag)“ als unzulässig bewertet, unter anderem weil der Button „erst recht nicht ausschließlich“ mit den Worten ,zahlungspflichtig bestellen“ beschriftet war. ${ }^{22}$ Neben der korrekten Beschrif-

\footnotetext{
16 Tendiert wohl zur Zulässigkeit Alexander, NJW 2012, 1985, 1988 f.

17 Dazu unter II. 1. b).

18 So etwa Wicker, MMR 2012, 783.

19 So auch Weidenkaff, in: Palandt (Fn. 8), § 598 BGB, Rn. 3.

20 So wohl auch Trusted Shops, Die Button-Lösung, Köln, http://www.trustedshops.de/shop-info/wpcontent/uploads/2012/03/120320_Whitepaper-Button-L\%C3\%B6sung.pdf, letzter Abruf am 9.8.2013, S. 9; Müller, K\&R 2012, 791, 792 zu ,sukzessive(n) Multibutton“.

21 BT-Drs. 17/7745, S. 12.

22 LG Berlin, Urteil vom 17.7.2013, AZ: 97 O 5/13.
} 
tung der Schaltfläche dürfen auch keine ablenkenden oder irreführenden Gestaltungselemente abgebildet sein.

\section{b) Formale Gestaltung der Schaltfläche}

$\S 312 \mathrm{~g}$ Abs. 3 Satz 2 BGB fordert zudem, dass der Schriftzug gut lesbar sein muss. Anforderungen an die formale Gestaltung werden in der Literatur jedoch vor allem im Zusammenhang mit den Informationspflichten aus Abs. 2 diskutiert. ${ }^{23}$ Jedoch führt nur die fehlerhafte Gestaltung der Schaltfläche dazu, dass der Vertrag nicht zustande kommt. Zum Teil wird (indirekt) auf die Ausführungen zu Abs. 2 verwiesen. ${ }^{24}$ Explizit wird etwa darauf hingewiesen, dass Voraussetzung für die gute Lesbarkeit der Schaltflächenbeschriftung ist, dass sie „,bei üblicher Bildschirmauflösung gut erkennbar" ${ }^{25}$ die Schrift also nicht zu klein oder kontrastarm ${ }^{26}$ ist.

\section{aa) Gute Lesbarkeit}

Zur Konkretisierung der guten Lesbarkeit bietet sich ein Rückgriff auf Ausführungen zu anderen Vorschriften an. In Betracht kommt das Merkmal der leichten Erkennbarkeit der Anbieterkennzeichnung nach $\S 5$ Abs. 1 HS 1 TMG, das der Preisklarheit des $\S 1$ Abs. 6 PAngV und das der zumutbaren Kenntnisnahme von Allgemeinen Geschäftsbedingungen nach $\S 305$ Abs. 2 Nr. 2 BGB. ${ }^{27}$ Außerdem sind die Pflichtinformationen nach $\S 312 \mathrm{c}$ Abs. 1 BGB i.V.m. Art. $246 \S 1$ Abs. 1 HS 1 EGBGB sowie nach $\S 312$ g Abs. 1 Nr. 2 BGB i.V.m. Art. $246 \S 3$ EGBGB „klar und verständlich“" anzugeben. Erstere müssen zudem auch „,in einer dem eingesetzten Fernkommunikationsmittel entsprechenden Weise" angegeben werden. Alle Vorschriften behandeln die formale Gestaltung von Informationen, die der Diensteanbieter seinen Kunden in seinem Online-Shop mitteilen muss.

Insgesamt wird bei der Frage der formalen Gestaltung auf die Faktoren Schriftgröße, -art und -farbe sowie Hintergrundfarbe und damit Kontrast abgestellt. ${ }^{28}$ Nach dem Urteil des LG Leipzig ist es beispielsweise nicht ausreichend, wenn die Beschriftung, die über die Zahlungspflicht aufklären soll, etwa nur, ,erheblich kleiner und dünner geschrieben und (...) bei kleinen Bildschirmen fast nicht lesbar (ist) und (...) auch ansonsten aufgrund des Größenverhältnisses leicht übersehen werden (kann). “29

Es wird grundsätzlich von einem Verbraucher erwartet werden können, dass er die Schaltflächenbeschriftung lesen kann, wenn diese so formatiert ist, wie die übrigen Inhalte der Seite. Nur in seltenen Fällen, in denen die Formatierung der Seite insgesamt auffällig vom Standard abweicht (etwa besonders klein und wenig kontrastreich ist), wird eine abstrakte Beurteilung der Lesbarkeit nötig sein.

\section{bb) Nähe zu Pflichtinformationen}

Auch aus dem neuen $\S 312 \mathrm{~g}$ Abs. 2 Satz 1 BGB ergibt sich im Umkehrschluss eine formale Gestaltungsanforderung an die Schaltfläche. Die in Abs. 2 genannten Informationen sind in räumlicher Nähe zur Schaltfläche zu platzieren. ${ }^{30}$ Die Schaltfläche muss deshalb bei üblicher

23 BT-Drs. 17/7745, S. 10 f.; Raue, MMR 2012, 438, 441.

24 So verweist Kredig/Uffmann, ZRP 2011, 36, 39 etwa auf BT-Drs. 17/7745, S. 11; Roth, VuR 2012, 477, 481 trennt nicht zwischen den gestalterischen Anforderungen der verschiedenen Absätze.

25 Leier, CR 2012, 378, 383.

26 Grüneberg, in: Palandt (Fn. 8), §312g BGB, Rn. 14.

27 Dazu zählt bereits nach BT-Drs. 7/3919, S. 18 auch die mühelose Lesbarkeit.

28 Etwa Micklitz/Schirmbacher, in: Spindler/Schuster, Recht der elektronischen Medien, 2. Aufl. 2011, § 312c BGB, Rn. 31 m.w.N; Brönneke, in: Roßnagel, Recht der Telemediendienste, 2013, § 5 TMG, Rn. 82; Thamm/Detzer, BB 1989, 1133; Köhler, in: Köhler/Bornkamm, UWG, 30. Aufl. 2012, § 1 PAngV, Rn. 49. So auch Thüsing, in: Staudinger, BGB, 15. Aufl. 2011, Buch 2, § 312g BGB, Rn. 68.

29 LG Leipzig, Urteil vom 26.7.2013, AZ: 08 O 3495/12.

30 Bierekoven, ITRB 2012, 186, 187; Raue, MMR 2012, 438, 438. 
Bildschirmauflösung gleichzeitig mit den Informationen zu sehen sein. ${ }^{31}$ Nicht schädlich ist, wenn nicht alle Informationen auf einen Blick erfasst werden können und es deshalb nötig ist, innerhalb der Informationen zu scrollen. ${ }^{32}$ Allerdings dürfen die Angaben nicht erst unter dieser angebracht sein, da der Schaltfläche eine (Vertrags-)Abschlussfunktion zukommt und der Verbraucher unterhalb dieser keine relevanten Informationen mehr erwartet. ${ }^{33}$ Daraus ergibt sich, dass zumindest die zuletzt genannten Informationen bei üblicher Bildschirmauflösung über der Schaltfläche zu sehen sein müssen.

\section{Frühere und aktuelle Rechtslage}

Durch einen Vergleich zwischen der früheren und der aktuellen Rechtslage werden die Konsequenzen der Gesetzesänderung deutlich.

\section{a) Seriöse Anbieter}

Besuchte der Verbraucher eine Webseite, weil er dort mit einem Unternehmer einen kostenpflichtigen Vertrag über die Lieferung von Waren oder Erbringung von Dienstleistungen abschließen möchte, konnte er dies bisher ohne weiteres tun. Zwar bestanden verschiedene Pflichten für den Unternehmer, ihre Einhaltung hatte jedoch keine Auswirkung auf den Vertragsschluss. Verstößt ein Unternehmer nunmehr jedoch gegen die Vorschriften des neuen $\S 312 \mathrm{~g}$ Abs. 3 BGB, kommt ein kostenpflichtiger Vertrag nach Abs. 4 nicht zustande.

\section{b) Unseriöse Anbieter}

Anders gestaltet sich die Situation, wenn der Verbraucher eine Webseite besucht, ohne dass er einen kostenpflichtigen Vertrag abschließen möchte, beispielsweise, weil er anscheinend kostenlose Informationen sichten möchte. In der Vergangenheit kam es immer wieder dazu, dass Verbraucher nach dem Besuch einer solchen Webseite eine Rechnung erhielten. Regelmäßig berief sich der Anbieter der jeweiligen Kostenfalle auf einen angeblichen Vertragsschluss, da - optisch schwierig auf der Webseite zu entdecken - auf die Kostenpflichtigkeit des Angebots hingewiesen wurde. Viele Verbraucher zahlten diese Rechnungen aus Unsicherheit, aufgrund des Drucks und der Kosten-Aufwand-Relation. ${ }^{34}$

Aus rechtlicher Sicht waren sie zur Zahlung aber regelmäßig nicht verpflichtet, weil es am Rechtsbindungswillen des Verbrauchers mangelte, der mit der Anmeldung keinen kostenpflichtigen Vertrag abschließen wollte, was dem Unternehmer auch bekannt war. Außerdem konnte der Vertrag, falls er doch geschlossen wurde, regelmäßig wegen arglistiger Täuschung angefochten werden. ${ }^{35}$ Auch nach der neuen Rechtslage ist der Verbraucher nicht gegen seinen Willen an den Vertrag gebunden. Es liegt somit lediglich ein weiterer Grund dafür vor, dass der Verbraucher nicht zur Zahlung verpflichtet ist. ${ }^{36}$

\section{c) Zwischenfazit}

Allerdings konnten die früheren Instrumente den Verbraucher rein faktisch nicht ausreichend schützen. ${ }^{37}$ Dies gilt zumindest für die Verbraucher, die sich keine Rechtsberatung gesucht haben oder selbst nicht ausreichend über die Rechtslage aufgeklärt waren. Die Button-Lösung

31 BT-Drs. 17/7745, S. $10 \mathrm{f}$.

32 BT-Drs. 17/8805, S. 6; anders noch BT-Drs. 17/7745, S. 11.

33 Raue, MMR 2012, 438, 441; Bergt, NJW 2012, 3541, 3542.

34 Detailliert im Einzelnen Zander, Deutscher Verbraucherschutz im E-Commerce am Beispiel von Abonnement- bzw. Vertragsfallen im Internet, Dissertation Univ. Kassel, 2012, S. 5 f. m.w.N.

35 Ausführlich Blasek, GRUR 2010, 396, 396 m.w.N.; Kredig/Uffmann, ZRP 2011, 36; Buchmann/Majer/Hertfelder/Vögelein, NJW 2009, 3189; zum Teil a.A. Zander (Fn. 34), S. 85 ff.

36 Anstelle vieler Buchmann/Majer, K\&R 2010, 635, 636 f.; Wilschke, VuR 2012, 171, 173 ff.; Tamm, VuR 2012, 217, $218 \mathrm{ff}$.

37 So auch Roth, VuR 2012, 477, 479. 
soll genau diese Gruppe von Verbrauchern unterstützen, indem keine komplexe Auslegung mehr nötig ist, sondern eine klare und leicht verständliche Regelung existiert, die es auch juristischen Laien ermöglicht, die Situation rechtlich einzuordnen. ${ }^{38}$

\section{Ungelöste Probleme}

Um das Ziel der Norm zu erreichen, das heißt, dem einzelnen Verbraucher leicht zu erkennen zu geben, ob eine Zahlungspflicht besteht oder nicht, reicht die Button-Lösung allein nicht.

\section{Generelle Unkenntnis der Verbraucher}

Entscheidend für das Erkennen der Zahlungspflicht ist das Bewusstsein des Verbrauchers darüber, dass ohne eine rechtskonform gestaltete Schaltfläche kein Vertrag zustande kommt. Bereits die frühere Rechtslage reichte grundsätzlich aus, um die Zahlung an Kostenfallen zu verweigern. Dass Verbraucher trotzdem zahlten, lag v. a. an der Unkenntnis der Rechtslage.

Es ist nicht damit zu rechnen, dass alle Betreiber von Kostenfallen aufgrund der Gesetzesänderung aufhören, Rechnungen in der Hoffnung zu verschicken, dass sie zumindest ein Teil der Verbraucher zahlt. Nur wenn die Verbraucher also über die neue Rechtslage informiert sind, werden sie sich darauf berufen, dass sie keine gesetzeskonforme Schaltfläche angeklickt haben und deshalb auch nicht an den Vertrag gebunden sind. ${ }^{39}$ Zwar hat die Einführung des $\S 312 \mathrm{~g}$ BGB zu einer gewissen Berichterstattung in den Medien geführt. Zu einer breiten und ausführlichen Bekanntmachung der neuen Rechtslage und der Handlungsmöglichkeiten betroffener Verbraucher kam es allerdings nicht. Auch vorher gab es bereits Berichterstattungen über die Rechtslage bei Kostenfallen, ohne dass dadurch wesentlich verhindert wurde, dass Verbraucher unberechtigte Rechnungen beglichen.

\section{Unkenntnis der Verbraucher über konkrete Umsetzung}

Selbst bei Kenntnis der Button-Lösung muss sich der Verbraucher der Rechtslage so sicher sein, dass er sich - trotz zum Teil massiver Einschüchterungsversuche - auf sein Wissen verlässt und darauf beruft. ${ }^{40}$ Im Normalfall wird ein Verbraucher jedoch nicht alle problematischen Gestaltungsformen richtig beurteilen können. Einige Betreiber von Kostenfallen werden vermutlich versuchen, ihre Webseite an die neuen Vorschriften anzupassen, ohne dass der Verbraucher die Informationen tatsächlich zur Kenntnis nimmt. ${ }^{41}$ Gerade diese werden gezielt eine unklare Gestaltung, wie die (unzulässige) Beschriftung ,mieten“, wählen. ${ }^{42}$

Diese Möglichkeit bietet sich ihm, da der Gesetzestext zwar einen konkreten Wortlaut (,zahlungspflichtig bestellen“) vorschlägt, diesen allerdings nicht vorschreibt. ${ }^{43}$ Wenn der Anbieter also die Beschriftung anders als vorgeschlagen umsetzt, kann ein durchschnittlicher Verbraucher nicht unbedingt erkennen, ob damit die gesetzlichen Anforderungen erfüllt werden. Der Verbraucher könnte dann bei Erhalt einer Rechnung zum Schluss kommen, dass er zur Zahlung verpflichtet ist. Bei nur oberflächlicher Kenntnis der gesetzlichen Vorgaben könnte er auch (fälschlicher Weise) davon ausgehen, dass jede andere Formulierung als ,zahlungspflichtig bestellen" unwirksam ist.

Untersuchungen zur Wahrnehmung von Informationen auf Webseiten haben etwa für Sicherheitssiegel gezeigt, dass Verbraucher diesen übermäßig hohe Garantien zusprechen, die nicht

\footnotetext{
38 Vgl. BT-Drs. 17/7745, S. 6; Tamm, VuR 2012, 217, 220; Roth, VuR 2012, 477, 479; kritisch Ernst, VuR 2012, 205, 206.

39 Blasek, GRUR 2010, 396, 402; Borges, BB 2011, I; Roth, VuR 2012, 477, 482

$40 \quad$ Blasek, GRUR 2010, 396, 401 f.; Leier, CR 2012, 378, 384.

$41 \quad$ Kredig/Uffmann, ZRP 2011, 36, 39.

42 Eine Vielzahl anderer Varianten ist vorstellbar, so auch Kredig/Uffmann, ZRP 2011, 36, 39.

43 Kritisch dazu Alexander, NJW 2012, 1985, 1988; Zander (Fn. 34), S. 371.
} 
der Realität entsprechen. ${ }^{44}$ In gleicher Weise kann erwartet werden, dass die Bedeutung der Button-Lösung, insbesondere die rechtliche Relevanz, für Verbraucher unklar bleibt.

\section{Nachträgliches Nachvollziehen und Nachgeben}

Trifft beim Verbraucher heute eine Rechnung von einem Kostenfallenbetreiber ein, muss er, um den unberechtigten Forderungen nicht nachzugeben, in diesem Moment nachvollziehen (oder mit großer Sicherheit wissen), ob auf der entsprechenden Schaltfläche der Hinweis auf die Zahlungspflicht fehlte. Empirische Studien zum Verhalten von Verbrauchern im Internet zeigen allerdings, dass Hinweise in Webseiten häufig übersehen werden. ${ }^{45}$ Das ist insbesondere der Fall, wenn der Verbraucher ein bestimmtes Ziel verfolgt (etwa Informationen zu erhalten), und nicht primär darauf aus ist, unberechtigte Zahlungsforderungen zu verhindern.

Hinzu kommt, dass der Verbraucher dies rückblickend wissen muss. Dazu muss er sich an die konkrete Gestaltung der Webseite bei dem ursprünglichen Besuch erinnern - und daran, dass er den Bezahl-Button ${ }^{46}$ nicht gedrückt hat. ${ }^{47}$ Besonders schwierig ist dabei, dass sich der Verbraucher vielleicht bewusst daran erinnern kann, wenn er eine rechtskonforme Schaltfläche gesehen oder sogar gedrückt hat. ${ }^{48}$ Handelt es sich jedoch um eine Schaltfläche, die nicht eindeutig den gesetzlichen Anforderungen entspricht und vom Verbraucher deshalb - wie vom unseriösen Diensteanbieter gewollt - nicht als solche erkannt wurde, wird er sich kaum daran erinnern, da er diesem Anklicken keine besondere Bedeutung zugemessen hat. Genau in diesem Falle wäre es jedoch wichtig, die konkrete Gestaltung zu dem Zeitpunkt nachzuvollziehen. ${ }^{49}$

Das gilt auch für den Fall, dass nur behauptet wird, der Verbraucher habe auf eine gesetzeskonform gestaltete Schaltfläche gedrückt, wenn sich der Verbraucher nach einigen Wochen nicht mehr genau daran erinnert. ${ }^{50}$ Verbraucher werden also höchstwahrscheinlich weiterhin unberechtigte Rechnungen begleichen, da sie weiterhin dem Druck der unseriösen Anbieter, etwa in Form von (anwaltlichen) Zahlungsanforderungen, ausgesetzt sind.

\section{Technische Unterstützung beim Umgang mit (vermeintlichen) Bezahl-Buttons}

Trotz der Einführung der verbraucherschutzfreundlichen Button-Lösung bestehen weiterhin verschiedene, je nach Verbraucher auch unterschiedliche Probleme. Dazu führt vor alle die Tatsache, dass die Button-Lösung im Bewusstsein der Verbraucher noch nicht ausreichend verankert ist. Gerade für unseriöse Anbieter besteht auch immer noch ein Anreiz, die Zahlungspflicht raffiniert zu verschleiern, um weiterhin von Kostenfallen zu profitieren. Im Folgenden wird deshalb untersucht, ob und inwieweit der Verbraucher technisch unterstützt werden kann, wo das (geltende) Recht ungelöste Probleme hinterlässt.

Eine technische Unterstützung zum Erkennen eines (vermeintlichen) Bezahl-Buttons kann erreicht werden, soweit eine Software die rechtlichen Anforderungen an die Button-Lösung überprüfen kann. Die Unterstützung wird möglichst umfassend, wenn sie unabhängig von einer Mitwirkung jedes einzelnen Anbieters ist und auf dem Gerät des Nutzers läuft, sodass

44 Kirlappos/Sasse/Harvey, Why Trust Seals Don't Work, A Study of User Perceptions and Behavior, in: Katzenbeisser et al., Lecture Notes in Computer Science 2012, S. 308.

45 Z.B. für Authentisierungsbilder beim Login in Schechter, et al., The Emperors New Security Indicators, IEEE Security \& Privacy, 2007.

46 Im Folgenden wird die in $\S 312 \mathrm{~g}$ Abs. 3 Satz 2 BGB benannte Schaltfläche zur Unterscheidung zu ,einfachen“ Buttons als „Bezahl-Button“ bezeichnet.

47 So auch Buchmann/Majer, K\&R 2010, 635, 637.

48 Roth, VuR 2012, 477, 481; a.A. Kredig/Uffmann ZRP 2011, 36, 39.

49 Roth, VuR 2012, 477, 481 bezieht sich jedoch auf die Erinnerung an den korrekt gestalteten Bezahl-Button.

$50 \quad$ Vgl. Buchmann/Majer, K\&R 2010, 635, 637. 
sie den Verbraucher bei jedem seiner Schritte begleiten kann. Eine solche Software könnte beispielsweise vom Bundesministerium für Verbraucherschutz zur Verfügung gestellt werden. $^{51}$

Dabei würde eine ideale Unterstützung an drei verschiedenen Zeitpunkten ansetzen. Zunächst sollte die Software den Verbraucher dabei unterstützen, ein zahlungspflichtiges Angebot zu erkennen, damit gar keine Basis für eine spätere Zahlungsforderung entsteht. Dann sollte der Verbraucher auch in dem Moment unterstützt werden, wenn er mit ungerechtfertigten Forderungen unseriöser Anbieter konfrontiert wird. Zuletzt ist auch eine langfristige Unterstützung in Form einer Sensibilisierung und Aufklärung des Verbrauchers durch die Software förderlich, damit sich der Verbraucher - auch unabhängig von der technischen Unterstützung im Einzelfall - der Rechtslage bewusst ist.

\section{Möglichkeiten zum automatisierten Erkennen kostenpflichtiger Angebote}

Um den Verbraucher beim Umgang mit unseriösen Anbietern zu unterstützen, muss die Software zunächst in der Lage sein, ein kostenpflichtiges Angebot zu erkennen, um dann auf den (potentiellen) Abschluss eines Vertrags hinzuweisen, der eine Zahlungspflicht begründet. Um kostenpflichtige Angebote im Internet zu erkennen, wurde die Button-Lösung eingeführt. Die Software könnte den Verbraucher deshalb am effektivsten unterstützen, wenn sie aufgrund eindeutiger gesetzlicher Vorgaben erkennen könnte, ob ein rechtskonform gestalteter BezahlButton vorliegt.

\section{a) Möglichkeiten zum automatisierten Erkennen des Bezahl-Buttons}

Um einen rechtskonformen Bezahl-Button als solches zu erkennen, bietet es sich zunächst an, automatisiert die Beschriftung von Buttons und Hyperlinks sowie Texte in unmittelbarer Nähe zu Checkboxen zu prüfen. Per Texterkennung kann automatisiert ausgelesen werden, ob auf einer Unterseite einer Webseite der Wortlaut des Gesetzestextes ,zahlungspflichtig bestellen“ in Text oder - mit Einschränkungen ${ }^{52}$ - in Grafik verwendet wird. Soweit die Software eine entsprechende Beschriftung erkennt, ist auch der Rückschluss zulässig, dass mit Betätigen der Schaltfläche ein Vertrag geschlossen werden soll, der eine Zahlungspflicht begründet, da nur auf einer Schaltfläche mit dieser Funktion eine solche Beschriftung zulässig ist.

Allerdings sieht das Gesetz gerade diese Beschriftung nicht als verpflichtend an. Vielmehr kann der Unternehmer auch andere Formulierungen nutzen. ${ }^{53}$ Technisch ist es möglich, zusätzlich andere Formulierungen, wie die der Gesetzesbegründung und die in der Literatur genannten, zu erkennen. Dazu ist es lediglich nötig, eine Liste von zulässigen Begriffen anzulegen. Dabei besteht auch die Möglichkeit, nach Kombinationen zu suchen. Eine solche Liste kann jedoch nicht den Anspruch der Vollständigkeit erheben, solange keine abschließende Liste zulässiger Formulierungen existiert. Durch regelmäßige Aktualisierung der Liste, etwa aufgrund neuer Literatur oder Gerichtsentscheidungen, wird allerdings eine hohe Trefferquote möglich sein, sodass gute Ergebnisse erreicht werden können. Eine besonders umfangreiche, aktuelle und damit effektive Liste entstünde, wenn die Verbraucherschutzverbände die Aktua-

51 Im Rahmen des Forschungsprojekts „Benutzerunterstützung zur Bewertung der Vertrauenswürdigkeit von Webseiten und Webshops (Internet Usage Support: InUse)“ wird in interdisziplinärer Zusammenarbeit unter anderem von den Autoren ein Prototyp einer solchen Software entwickelt. Der Beitrag entstand im Rahmen des Forschungsprojekts. Das Vorhaben wird aus Mitteln des Bundesministeriums für Ernährung, Landwirtschaft und Verbraucherschutz (BMELV) aufgrund eines Beschlusses des deutschen Bundestages gefördert. Die Projektträgerschaft erfolgt über die Bundesanstalt für Landwirtschaft und Ernährung (BLE) im Rahmen des Programms zur Innovationsförderung.

52 Für die Erkennung von Text in Bildern wird die „Optical Character Recognition“ eingesetzt, die allerdings keine umfassende Erkennung garantieren kann. Vgl. Rice/Nagy/Nartker, Optical Character Recognition, An Illustrated Guide to the Frontier, 1999.

53 Detailliert dazu unter ,a) Beschriftung der Schaltfläche“. Kritisch dazu auch Alexander, NJW 2012, 1985, 1988; Zander (Fn. 34), S. 371. 
lisierung übernehmen würde. Die Verbraucherschutzverbände sind regelmäßig die ersten Ansprechpartner für ratsuchende Verbraucher und erfahren somit sehr schnell von neuen Gestaltungsformen von Kostenfallen. Damit haben sie die Möglichkeit die Liste mit Formulierungen, bei denen der Verbraucher auf die mögliche Zahlungspflichtigkeit hingewiesen werden sollte, auf einem sehr aktuellen Stand zu erhalten. Je länger diese Liste gepflegt würde, desto vollständiger würde sie. So könnte es nur noch in einigen wenigen Fällen dazu kommen, dass nicht jede rechtskonform beschriftete Schaltfläche als solche erkannt wird.

\section{b) Optimieren des automatisierten Erkennens des Bezahl-Buttons}

Auch wenn vielen Verbrauchern durch eine solche Software geholfen werden kann, bietet diese Analyse also keine absolute Sicherheit. ${ }^{54}$ In der Literatur wurde deshalb auch vorgeschlagen, dass zumindest der Begriff „zahlungspflichtig“ festgeschrieben werden sollte und nur das Verb abweichen darf, um entsprechend an das Angebot angepasst zu werden. ${ }^{55}$ Durch eine solche Regelung wäre auch das automatisierte Auslesen möglich.

Alternativ könnte auch eine abschließende Liste mit zulässigen Beschriftungen verbindlich festgelegt werden. Diese könnte beispielsweise lediglich die im Gesetz und in der Gesetzesbegründung genannten Begriffe und Phrasen umfassen. Diese Liste könnte, falls nötig, auch aktualisiert werden. Zulässig wäre aber immer nur die Verwendung der in der jeweils aktuellen Liste genannten Formulierungen.

Anstelle einer Liste mit zulässigen Formulierungen könnte auch eine Pflicht zur Aufnahme bestimmter Metadaten in den Quelltext der Webseite eingeführt werden, die das Vorhandensein eines Bezahl-Buttons anzeigen und als solche von der Software erkannt werden können. Durch eine solche Pflicht wäre ein einfacheres und vor allem zuverlässigeres softwaretechnisches Erkennen möglich, da Fehlerquellen bei der Erkennung von Texten auf Schaltflächen vermieden würden. Jedoch wäre es Verbrauchern ohne die Softwareunterstützung nicht möglich, die Metadaten auszulesen.

Bei der Abwägung zwischen Sichtbarkeit und technischer Zuverlässigkeit muss zugunsten der Sichtbarkeit für alle Verbraucher entschieden werden, da keine Pflicht zur standardmäßigen Einbindung entsprechender Software in die Basisversion von Browsern besteht. Eine andersartige gesetzliche Lösung würden nur die Verbraucher unterstützen, die bereits sensibilisiert sind und deshalb die Software installiert haben. Insoweit wäre diese Alternative aus verbraucherschutzrechtlicher Sicht weniger wünschenswert. Ideal wäre eine Kombination aus einem eindeutig formulierten Hinweis, der für den Verbraucher sichtbar ist, und der technischen Markierung zur softwaretechnischen, zuverlässigen Auswertung, um etwa auch den Abschluss von Verträgen im Internet, die eine Zahlungspflicht begründen, technisch auszuschließen.

\section{c) Automatisiertes Erkennen der Rechtskonformität der Beschriftung}

$\S 312 \mathrm{~g}$ Abs. 3 Satz 2 BGB macht bezüglich der Beschriftung nicht nur Vorgaben zur Formulierung, sondern auch zum Umfang. Neben den zuvor besprochenen Formulierungen darf nichts anderes auf der Schaltfläche stehen. Für die im Rahmen der Liste erfassten zulässigen Formulierungen kann automatisiert ausgelesen werden, wenn unerlaubterweise weitere Texte auf der Schaltfläche stehen. Zwar kommt in diesem Fall nach § 312g Abs. 4 BGB kein Vertrag zustande. Der Verbraucher sollte aber dennoch auf die Schaltfläche hingewiesen werden, um zu vermeiden, dass der Verbraucher in unnötige Streitigkeiten mit einem KostenfallenBetreiber verwickelt wird, weil er die wesentliche Information, nämlich die entstehende Zah-

$54 \mathrm{Zu}$ bedenken ist, dass der Anbieter einer solchen Software unter Umständen für seine Software und ihre (insbesondere möglicherweise fehlerhaften) Ergebnisse gegenüber dem Nutzer und dem Anbieter eines bewerteten Webshops haftet. Diese Frage ist detailliert unter genauer Berücksichtigung der Funktionen und Anzeigen der Software zu untersuchen. Dies ist im Rahmen dieses Beitrags nicht möglich.

$55 \quad$ Zander (Fn. 34), S. 372. 
lungspflicht, übersehen hat. Die Formulierung und/oder Gestaltung des Hinweises auf einen solchen vermeintlichen Bezahl-Button muss jedoch anders gestaltet werden als bei einer rechtskonform gestalteten Schaltfläche.

Dies gilt auch, soweit nicht ausreichend eindeutige Beschriftungen wie „mieten“ ohne klärende Zusätze erkannt werden. Denn zur Unterstützung des Verbrauchers müssen vor allem auch solche Schaltflächen erkannt werden, die nicht eindeutig den gesetzlichen Anforderungen entsprechen und eindeutig auf die Kostenpflicht hinweisen. Neben dem zuvor genannten Grund wurde auch festgestellt, dass die gesetzlichen Bestimmungen nicht konkret genug sind, um mit Sicherheit alle rechtskonform gestalteten Schaltflächen zu erfassen, sodass ein Verbraucher auch auf solche Schaltflächen hingewiesen werden muss, die nur einen Teil der gesetzlichen Vorgaben beachten. Denn gerade bei diesen Schaltflächen rechnet der Verbraucher unter Umständen nicht mit dem Abschluss eines Vertrags, der eine Zahlungspflicht begründet.

\section{d) Automatisiertes Erkennen der Rechtskonformität der Gestaltung}

Um den Verbraucher auch darüber zu informieren, wenn Schaltflächen die rechtlichen Anforderungen „knapp“ verfehlen, muss auch die formale Gestaltung der Schaltfläche geprüft werden. Schriftgröße, Schriftart, Farbe und Kontrast sind durch die Software - vergleichbar mit automatisierten Accessibility-Prüfungen ${ }^{56}$ - automatisiert auszulesen. Diese Werte können dann mit Standardwerten verglichen werden, sodass eine Aussage über die abstrakte Lesbarkeit getroffen werden kann. Zudem können die Werte auch mit denen der übrigen Inhalte der Unterseite verglichen werden.

\section{e) Zwischenfazit}

Solange aber nicht jede rechtskonforme Schaltfläche als solche erkannt werden kann, ist es nicht möglich, etwa den Abschluss von Verträgen im Internet, die eine Zahlungspflicht begründen, technisch auszuschließen, auch wenn dies vom Nutzer gewünscht ist. So kann also technisch nicht verhindert werden, dass aus Versehen auf den Button gedrückt wird oder Rechtsstreitigkeiten aufgrund von Handlungen durch Kinder entstehen. ${ }^{57}$

\section{f) Weitere Erkennungsmöglichkeit von kostenpflichtigen Webseiten}

Die Button-Lösung wurde mit dem Ziel eingeführt, dass der Verbraucher ohne weiteres erkennt, wenn er im Internet einen Vertrag abschließt, der eine Zahlungspflicht begründet. Wie festgestellt wurde, ist es dem Verbraucher jedoch nicht immer möglich, die Schaltfläche zu erkennen, mit der ein solcher Vertrag abgeschlossen werden soll. Da dies auch mit softwaretechnischer Unterstützung nicht immer möglich ist, sollten auch andere Merkmale als der Bezahl-Button selbst herangezogen werden. Nach § 312g Abs. 2 Satz 1 BGB i.V.m. Art. $246 \S 1$ Abs. 1 Nr. 7 EGBGB muss, unmittelbar bevor der Verbraucher seine Bestellung abgibt, klar und verständlich in hervorgehobener Weise der Preis der Waren genannt werden. Durch Texterkennung kann nach Angaben mit dem Zusatz $€$, Euro oder EUR gesucht werden.

Dies ist eine einfache und wenig aufwendige Methode, um eine ergänzende Absicherung der Ergebnisse der Button-Analyse ${ }^{58}$ zu erhalten. Soweit solche Preisangaben auf einer Unterseite zusammen mit einer Schaltfläche mit ähnlicher Formulierung wie in der Liste zu finden sind, sollte die Software auch diese als potentiell kostenpflichtige Webseite ansehen.

56 Accessibility-Prüfungen werden auf Webseiten eingesetzt, um zu prüfen, ob auch benachteiligte Besucher, etwa mit Sehschwäche, die Seiten lesen können. Zu automatisierten Prüfungen vgl. W3C, Web Accessibility Evaluation Tools, Overview. http://www.w3.org/WAI/RC/tools/, letzter Abruf am 9.8.2013.

57 Die Frage der rechtlichen Verbindlichkeit einer solchen Erklärung wird nicht betrachtet, da der Verbraucher (auch) vor unnötigen Belastungen wie Mahnschreiben der Kostenfallen-Betreiber geschützt werden soll.

58 Vgl. Abschnitt I.1. 


\section{Information des Nutzers}

Das reine Erkennen eines Bezahl-Buttons durch die Software ist für den Verbraucher nur dann von Nutzen, wenn dieser darüber informiert wird. Der Verbraucher muss deshalb durch einen Hinweis darauf aufmerksam gemacht werden, wenn sich auf der Unterseite, die er gerade besucht, ein Button zum Abschluss eines kostenpflichtigen Vertrages befindet. ${ }^{59}$

\section{a) Hinweis auf (vermeintlichen) Bezahl-Button}

Wurden ein (vermeintlicher) Bezahl-Button und Preisangaben auf einer Unterseite erkannt, ist der Verbraucher darauf hinzuweisen. Dazu kann beispielsweise die Preisangabe optisch hervorgehoben werden, wenn sie sich in der Nähe der Schaltfläche befindet, womit zumindest die Wahrscheinlichkeit stark erhöht wird, dass der Verbraucher diese Information zur Kenntnis nimmt. Ansonsten könnte die Information von der Software auch neben dem Button eingeblendet werden oder ein Pfeil darauf hinweisen, dass weiter oben, unten, rechts oder links auf der Seite Preisangaben gefunden und hervorgehoben wurden.

Dies könnte sich auch bei rechtskonform gestalteten Bezahl-Buttons als sinnvoll erweisen, um zu verhindern, dass etwa ein Teil der Kosten in den anderen Pflichtinformationen ,versteckt" wird. Der Verbraucher könnte sonst davon ausgehen, dass nur ein günstigerer Betrag zu zahlen ist und sich deshalb mit dem Abschluss des Vertrags einverstanden erklären. ${ }^{60}$

Auch wenn ein Bezahl-Button erkannt wird, der die gesetzlichen Anforderungen nur zum Teil erfüllt, muss der Verbraucher darauf hingewiesen werden. Obwohl dann kein Vertrag zustande kommt, handelt es sich gerade bei solchen Angeboten vermutlich um Kostenfallen, aufgrund derer der Verbraucher im Anschluss (unberechtigte) Zahlungsaufforderungen erhält. Dieser Hinweis sollte jedoch anders gestaltet werden, um zu verdeutlichen, dass es sich vermutlich um einen unseriösen Anbieter handelt.

\section{b) Auswirkung auf den Verbraucher}

Durch die Hinweise, die der Verbraucher beim Besuch von Webseiten, insbesondere von Webshops, erhält, wird der Verbraucher immer wieder über die Problematik informiert sowie dafür sensibilisiert und dadurch langfristig geschult. Dies wurde wiederholt als Voraussetzung für die verbraucherschützende Wirkung der Button-Lösung genannt, denn die Gefahr von Kostenfallen besteht vor allem deshalb fort, da weiterhin Aufmerksamkeit und Kenntnis der Verbraucher notwendig ist, um sie zu erkennen. ${ }^{61}$

Im Browser eingeblendete Hinweise mit ergänzenden Informationen können einen wichtigen Beitrag leisten, um das Bewusstsein für die Button-Lösung zu erhöhen. Voraussetzung dafür ist jedoch bereits ein bestehendes Problembewusstsein, das den Verbraucher zum Installieren der Software motiviert hat. Deshalb sind weiterhin flankierende Maßnahmen zur Schaffung des notwendigen Bewusstseins durch gezielte Informationskampagnen etwa in Schulen und Medien notwendig.

\section{Unterstützung in Konfliktsituationen}

Erhält der Verbraucher trotz der ihn begleitenden Software unberechtigte Zahlungsaufforderungen im Zusammenhang mit der Nutzung von Kostenfallen, kann die Software ihn darin bestärken, dem Druck des Kostenfallen-Betreibers Stand zu halten.

59 Zur Warnung vor der Eingabe von sensiblen Informationen werden in der Literatur etwa Popups neben den Eingabefeldern vorgeschlagen. Vgl. Maurer/De Luca/Kempe, Using data type based security alert dialogs to raise online security awareness, SOUPS 2011.

60 Auch Zander (Fn. 34), S. 371 befürchtet, dass Preisangaben zwischen anderen Pflichtangaben „,versteckt“ werden können.

61 So etwa BT-Drs. 17/7745, S. 6; Blasek, GRUR 2010, 396, 402; Borges, BB 2011, I. 


\section{a) Automatisiert erzeugter Screenshot}

Die Software könnte automatisiert einen Screenshot von jeder Seite machen und (verschlüsselt) auf dem Rechner des Nutzers speichern, wenn ein (vermeintlicher) Bezahl-Button betätigt wurde. Dadurch soll der Verbraucher vor allem im vorgerichtlichen Zeitraum unterstützt werden, da es bei Kostenfallen es in der Vergangenheit nur selten zu Gerichtsverfahren kam, u.a. weil die meisten Verbraucher die Rechnungen zahlten. ${ }^{62}$ Zwar ist es nicht möglich, alle getätigten Vertragsabschlüsse zu speichern, die eine Zahlungspflicht begründen, da nicht jeder Bezahl-Button mit Sicherheit erkannt wird. Deshalb ist der Umkehrschluss nicht zulässig, dass andere als die von der Software registrierten Abschlüsse nicht erfolgt sind. Der Verbraucher kann jedoch zumindest psychologisch in seiner Position bestärkt werden, sodass er weniger geneigt ist, dem Druck des Kostenfallen-Betreibers nachzugeben. Der Verbraucher erhält Zugriff auf Screenshots von allen Webseiten, auf denen ein als solcher (vermeintlicher) Bezahl-Button betätigt wurde und damit ein weitestgehend vollständiges Protokoll der Vertragsabschlüsse, die auf dem jeweiligen Gerät getätigt wurden.

\section{b) Manuell erzeugter Screenshot}

Zusätzlich besteht die Möglichkeit, dass der Verbraucher auf auffälligen Seiten selbst einen Screenshot machen und durch die Software speichern lässt. Damit erhält auch der in Bezug auf die Button-Lösung bereits informierte Verbraucher ein zusätzliches Instrument zur Unterstützung. Außerdem wäre es möglich, dass die Software registriert, wenn eine bestimmte Webseite häufiger manuell aufgrund einer verdächtigen Schaltfläche gemeldet wurde. Bestätigt sich der Verdacht, dass es sich um eine nicht rechtskonform gestaltete Schaltfläche handelt, könnten damit Schwächen der Software entdeckt und in der Folge optimiert werden.

\section{c) Auswirkung auf den Verbraucher}

Wenn die Webseite, auf der der Verbraucher angeblich den die Zahlungspflicht begründenden Vertrag abgeschlossen hat, nicht als Screenshot hinterlegt wurde, wird dies den Verbraucher zumindest darin bestärken, dass die ihn bei Vertragsabschluss treffende Zahlungspflicht nicht eindeutig kommuniziert wurde. Ist die Webseite hingegen dort hinterlegt, hat der Verbraucher die Möglichkeit, die Erscheinung der Webseite in dem Moment des Besuchs zu rekapitulieren.

Bei Bezahl-Buttons, die nach der automatisierten Prüfung die rechtlichen Anforderungen nicht ganz erfüllen, wird außerdem diese Information auch dem Screenshot beigefügt. Dabei darf der Screenshot selbst nur die vom Anbieter zur Verfügung gestellte Unterseite abbilden.

\section{d) Zwischenfazit}

Diese Unterstützung kann der Verbraucher genau in dem Moment abrufen, wenn er sich in einer Konfliktsituation befindet und sich unter Druck gesetzt fühlt, obwohl die kritische Situation vorüber ist, in der er vor der Entscheidung stand, ob er handelt oder nicht. Durch den Screenshot wird ein konkreter Bezug zu dem konkreten Fall hergestellt.

\section{Fazit}

Trotz des Ziels der Button-Lösung, den Verbraucher vor Kostenfallen im Internet zu schützen, bestehen weiterhin ungelöste Probleme. Eine entsprechend programmierte und beim Verbraucher installierte Software kann ihn zu drei verschiedenen Zeitpunkten unterstützen. Zunächst würde er in der konkreten Situation durch die Anzeige im Browser darauf aufmerk-

62 Im Rahmen dieses Beitrags wird nicht untersucht, welcher Beweiswert einem solchen Screenshot vor Gericht beigemessen werden kann Dabei wären auch technische Maßnahmen wie eine Verschlüsselung oder eine elektronische Signatur zu bedenken. 
sam gemacht, dass er auf einer gerade besuchten Webseite einen Vertrag abschließen kann, der für ihn eine Zahlungspflicht begründet. Werden ihm gegenüber im Nachhinein unberechtigte Zahlungsansprüche geltend gemacht, kann er zu diesem Zeitpunkt auf Screenshots der Webseiten zugreifen, auf denen ein Bezahl-Button betätigt wurde. Zuletzt erhält der Verbraucher eine langfristige Unterstützung, indem er durch die Hinweise der Software Einzelheiten erfährt und damit über die geltende Rechtslage informiert wird.

Eine vollständige Unterstützung ist jedoch nicht möglich, da der Gesetzgeber es unterlassen hat, die Beschriftung des Bestellbuttons oder eine Pflicht zum Hinterlegen von Informationen zu den Bezahl-Buttons im Quelltext der Webseiten festzulegen. Dies ist Folge der technikneutralen Formulierung der Norm. Auch die psychologischen Komponenten der Kostenfallen wurden im Rahmen der Gesetzgebung nicht ausreichend betrachtet. Für die Zukunft muss deshalb eine interdisziplinäre Zusammenarbeit zwischen Sicherheits-Informatikern, Verhaltenspsychologen und Juristen bei der Gestaltung von gesetzlichen Maßnahmen zum Verbraucherschutz im Internet gefordert werden.

Carina Boos, LL.M., ist wissenschaftliche Mitarbeiterin in der Projektgruppe verfassungsverträgliche Technikgestaltung (provet) im Forschungszentrum für InformationstechnikGestaltung (ITeG) der Universität Kassel bei Prof. Dr. Alexander Roßnagel.

Dr. Steffen Bartsch ist Post-Doc im Fachgebiet SecUSo (Security, Usability und Society) des Fachbereichs Informatik an der Technischen Universität Darmstadt.

Prof. Dr. Melanie Volkamer ist Juniorprofessorin am Fachbereich Informatik an der Technischen Universität Darmstadt. Sie leitet dort das Fachgebiet SecUSo (Security, Usability und Society). 